\title{
Psychotropic medication during endocrine treatment for breast cancer
}

\author{
Geertruida H. de Bock • Regina F. Musters • H. Jens Bos • Caroline P. Schröder • \\ Marian J. E. Mourits • Lolkje T. W. de Jong-van den Berg
}

Received: 4 March 2011 / Accepted: 24 July 2011 /Published online: 6 August 2011

(C) The Author(s) 2011. This article is published with open access at Springerlink.com

\begin{abstract}
Purpose Psychological problems are frequently mentioned in women treated for breast cancer in whom depression is mentioned as the most common disorder. The aim was to study the prescription of psychotropic medication in women with endocrine treatment for breast cancer in women in our prospective and consecutive pharmacy database.

Methods Women $(n=2,172)$ with at least one prescription of tamoxifen, fulvestrant, anastrazole, letrozole or exemestane were considered as breast cancer patients treated with endocrine therapy. This group was compared with an ageand family physician-matched group of women without cancer $(n=8,129)$, and the incidence risk ratio (IRR) and the $95 \%$ confidence intervals $(95 \% \mathrm{CI})$ were calculated. In addition, the prevalence of these psychotropic medication prescriptions and the $95 \% \mathrm{CI}$ were calculated.
\end{abstract}

\section{G. H. de Bock $(\bowtie)$}

Department of Epidemiology, University Medical Center Groningen, University of Groningen,

PO Box 30.001, 9700 RB, Groningen, The Netherlands

e-mail: g.h.de.bock@umcg.nl

R. F. Musters • H. J. Bos • L. T. W. de Jong-van den Berg Unit of PharmacoEpidemiology and PharmacoEconomics, Department of Pharmacy, University of Groningen,

Groningen, The Netherlands

\section{P. Schröder}

Department of Medical Oncology, University Medical Center Groningen, University of Groningen,

Groningen, The Netherlands

\section{J. E. Mourits}

Department of Gynaecology, University Medical Center

Groningen, University of Groningen,

Groningen, The Netherlands
Results There was an increased prescription of psychotropic medication in the female breast cancer patients on endocrine therapy: anxiolytics (IRR 2.07, 95\% CI 1.87-2.29), hypnotics and sedatives (IRR 2.59, 95\% CI 2.34-2.87) and antidepressants (IRR 1.46, 95\% CI 1.28-1.65). The prevalences of anxiolytics, hypnotics and sedatives were also increased in this group, indicating an increased use over time of these drugs. The prevalence of anti-depressant prescription was not increased, indicating short-term use only.

Conclusions This study indicated increased psychological distress due to breast cancer diagnosis and/or treatment in women on endocrine therapy. Anti-depressants were only prescribed for a short time. These data can contribute to an improved awareness of the impact of breast cancer (treatment) and therefore potentially to the optimizing of support for these patients.

Keywords Breast cancer survivor. Endocrine treatment. Psychotropic medication · Quality of life · Depression

\section{Introduction}

Breast cancer is the most prevalent type of cancer among women [1]. Endocrine therapy is an essential part of the adjuvant treatment for oestrogen receptor-positive and/or progesterone receptor-positive breast cancer [2]. This treatment exerts its effect by reducing the availability of oestrogen to micro-metastatic tumour cells. Meta-analyses have shown the efficacy of this treatment in improving disease-free and overall survival in women with early-stage as well as advanced breast cancer [3-7]. Current treatment standards involve $(\geq) 5$ years of endocrine therapy, including tamoxifen for premenopausal women and aromatase inhibitors for postmenopausal women. 
As the number of breast cancer patients (including younger women) treated for an extensive period of time is increasing, there is increased concern about long-term treatment-related symptoms and psychological well-being [8]. A better understanding of the psychosocial consequences of breast cancer on the quality of life after breast cancer can assist in recommendations for future treatment [9]. In a review including 125 references on psychosocial functioning of survivors of breast cancer, it is concluded that the majority of breast cancer survivors adjusted well 2 years since their first diagnosis. However, there is a subgroup of survivors reporting after-treatment psychological distress. The estimates for this group range from $5 \%$ to $50 \%$ [8].

We hypothesized that psychological distress accompanied with breast cancer diagnosis and/or the side effects of adjuvant therapy leads to an increased use of psychotropic medication in female breast cancer patients treated with endocrine therapy. Therefore, we assessed the use of psychotropic medication in female patients treated with endocrine therapy over time and compared the use of psychotropic medication with an age- and family physicianmatched cohort of women without cancer.

\section{Patients and methods}

\section{Drug prescription data}

The IADB is situated in the North of the Netherlands, covering an area of 3.4 million inhabitants. The IADB.nl is a pharmacy drug prescription database in the North of the Netherlands covering a population of 500,000 . All prescriptions for persons living in the IADB area are prospectively and consecutively entered into the pharmacy database. These prescriptions are, regardless of prescriber, insurance or reimbursement status, except over-the-counter (OTC) drugs and drugs dispensed during a hospital stay. So, all prescribed drugs (except in hospital use and OTC drugs) delivered to this population are available in the database. We have compared the drug use in our database with national prescription databases and found for all main drug groups comparable prevalences of drug use among sex and different age groups. Based on that, the IADB.nl can be considered as a database that is representative for the Netherlands regarding drug exposure. We have a methodological article in preparation on this topic.

Each prescription record comprises information about the drug, side effects, date of dispensing, amount dispensed, dose regimen and the prescribing physician. The indication for the prescription is not known. All drugs are coded according to the Anatomical Therapeutic Chemical (ATC) classification [11, 12]. For each patient, there are sex, date of birth, family physician and a unique, anonymous identifier. Due to a high patient pharmacy commitment in the Netherlands and sophisticated pharmacy software, the prescription records for each patient are virtually complete [13].

The role of the general practitioner

In the Netherlands, general practitioners hold a key position in health care. They are the first health care professionals to see people when they are ill, and they act as gatekeepers to secondary care. Most cases that present to a general practice are taken care of by the general practitioner alone. Sometime after primary treatment, the care for the patient is shifted back from hospital to general practice. When the care for the patient is shifted back to general practice, the general practitioner is responsible for the prescription of drugs. The most common indication for prescribing drugs in general practice is cardiovascular diseases, followed by diabetes mellitus and depression, respectively [14].

Females treated with endocrine therapy

In the Netherlands, endocrine and psychotropic medications are prescription drugs and cannot be obtained OTC. In the Netherlands, endocrine therapy is not indicated for the prevention of breast cancer, also not in high-risk women.

All women who were over 20 years of age during the study period 1994-2006 and had at least one prescription of anti-oestrogens (tamoxifen, fulvestrant) or aromatase inhibitors (anastrazole, letrozole, exemestane) were selected. Women with at least one prescription of anti-oestrogens or aromatase inhibitors were included in the analysis when there was information in the IADB database for a minimum of 180 days before the start date of adjuvant hormonal treatment. The analysis was based on these anti-oestrogens and aromatase inhibitors because these products are the preferred endocrine treatments for breast cancer in the Netherlands.

Women in the reference population

To compare the use of psychotropic medication among women using anti-oestrogens with the use of psychotropic medication among women not using anti-oestrogens, there were two options. Firstly, we could use the data of all women not using anti-oestrogens and correct for bias due to age and time in follow-up in the analysis. The second option was to draw a stratified sample of women not using anti-oestrogens from the cohort and correct in this way for bias due to age and time in follow-up. The reference population included women without any prescription of anti-oestrogens or aromatase inhibitors. In addition, women with any prescription of anti-neoplastic and immuno- 
modulating agents (ATC classification group L) were excluded. From this group, three to four women were matched for age at the start date of the anti-oestrogens or aromatase inhibitors prescription and family physician of the women in the index group. This start date was considered as the "theoretical" start date for women in the reference population. Women in the reference population could only be matched if there was information available in the IADB for at least 180 days before the theoretical start date. On average, there was a match of 3.7 reference women per woman with hormone receptor-positive breast cancer.

\section{Psychotropic medication}

Psychotropic medication was classified with the use of the following ATC codes: N05B (anxiolytics), N05C (hypnotics and sedatives) and N06A (anti-depressants).

\section{Analyses}

For females treated with endocrine therapy, the date of the first prescription of anti-oestrogens or aromatase inhibitors was considered as the starting date in the analysis. The women from the reference population received the starting date from the women with hormone receptor-positive breast cancer to whom they were matched.

To assess whether the start of psychotropic medication was increased, the cohort of female breast cancer survivors (index group, $n=2,172$ ) was compared with an age- and family physician-matched group of women without cancer (the reference population, $n=8,129$ ), and the incidence risk ratio (IRR) and the $95 \%$ confidence intervals $(95 \% \mathrm{CI})$ were calculated. The IRR was calculated as the incidence rate (IR) of the index group divided by the IR of the reference group [10]. For calculation of the IR, given the distribution of the data, 1 year before the starting date of adjuvant therapy was chosen as the starting point for follow-up. The number of patient days in the database was measured from this starting point for follow-up up until either the prescription date of psychotropic medication or the last known date of that person in the database. The incidence of drug prescribing was calculated as the number of incidence cases divided by the total number of patient days at risk. An incidence case was defined as a woman who did not receive a prescription for this medication for the last 180 days.

To assess the prescriptions of psychotropic medication over time, the half-year prevalence of these psychotropic medication prescriptions and the $95 \%$ CIs were calculated in the index as well as the reference group. The half-year prevalence was defined as the proportion of women having a psychotropic medication prescription during a half year and was calculated as the number of women who use psychotropic medication per 182 days (half year), divided by the total number of women present in that period. All statistical analyses were performed by Excel and SPSS 16.0. A $p$ value of less than 0.05 was considered to be statistically significant.

\section{Results}

The women in the study

In the IADB database, there were 2,172 female breast cancer patients treated with endocrine therapy. The reference group consisted of 8,129 women. The median age of the breast cancer patients treated with endocrine therapy was $64(20$ 93 ), with a median follow-up time of 2.6 years. The median age of women in the reference group was 62 (20-93), with a median follow-up time of 3.8 years (see Table 1). The number of women per year that started with tamoxifen, fulvestrant, anastrazole, letrozole and exemestane is shown in Table 2.

\section{Starting psychotropic medication}

There are significantly more starting prescriptions of psychotropic medication for female breast cancer patients treated with endocrine therapy when compared to women in the reference group (see Table 3). This increase is observed for all three groups of psychotropic medication, anxiolytics (IRR 2.07, 95\% CI 1.87-2.29), hypnotics and sedatives (IRR 2.59, 95\% CI 2.34-2.87) and anti-depressants (IRR $1.46,95 \%$ CI $1.28-1.65)$. Table 4 presents the specific drugs in the three psychotropic groups that were mostly prescribed. Of the 552 breast cancer patients who started anxiolytics, 313 received oxazepam. Of the 580 who started with hypnotics and sedatives, 383 received temazepam. Among the 331 who started an anti-depressant, 144 received amitriptyline, and $66(20 \%)$ received the combination paroxetine and tamoxifen (Table 4).

Psychotropic medication use

The prevalence of psychotropic medication use (anxiolytics, hypnotics and sedatives and anti-depressants) is presented in Fig. 1. The prevalence of psychotropic medication use (prevalence $0.45,95 \%$ CI $0.43-0.47$ ) is significantly increased among breast cancer patients using endocrine therapy when compared to the reference group (prevalence $0.32,95 \%$ CI $0.31-0.32$ ). In the period a half year after the start, there is still an increase of psychotropic medication use, although from this period, a decrease is observed. 
Table 1 Characteristics of women with hormone receptorpositive breast cancer in the index group and women in the reference group

${ }^{\text {a }}$ Index group - women with at least one prescription of tamoxifen, fulvestrant, anastrazole, letrozole or exemestane were considered as breast cancer patients treated with endocrine therapy

${ }^{\mathrm{b}}$ Reference group - an age- and family physician-matched group of women any prescription of tamoxifen, fulvestrant, anastrazole, letrozole or exemestane

${ }^{\mathrm{c}}$ Time of follow-up-time in observation

\begin{tabular}{|c|c|c|c|c|c|}
\hline & \multicolumn{3}{|c|}{ Index group ${ }^{\mathrm{a}}$} & \multicolumn{2}{|c|}{ Reference group ${ }^{b}$} \\
\hline Number & \multicolumn{3}{|l|}{2,172} & \multicolumn{2}{|l|}{8,129} \\
\hline $\begin{array}{l}\text { Mean number of reference } \\
\text { women per index woman }\end{array}$ & \multicolumn{3}{|l|}{1} & \multicolumn{2}{|l|}{3.7} \\
\hline Mean age at start date (SD) & \multicolumn{3}{|c|}{64 years (15 years) } & \multicolumn{2}{|c|}{63 years (15 years) } \\
\hline Median (range) & \multicolumn{3}{|c|}{64 years $(20-93)$} & \multicolumn{2}{|c|}{62 years $(20-93)$} \\
\hline \multirow{10}{*}{$\begin{array}{l}\text { Number of women } \\
\text { per age group }\end{array}$} & Age group & $n$ & $\%$ & $n$ & $\%$ \\
\hline & $20-30$ & 16 & 0.7 & 65 & 0.8 \\
\hline & $30-40$ & 94 & 4.3 & 387 & 4.8 \\
\hline & $40-50$ & 301 & 13.9 & 1,272 & 15.6 \\
\hline & $50-60$ & 487 & 22.4 & 1,969 & 24.2 \\
\hline & $60-70$ & 411 & 18.9 & 1,500 & 18.5 \\
\hline & $70-80$ & 444 & 20.4 & 1,652 & 20.3 \\
\hline & $80-90$ & 388 & 17.9 & 1,232 & 15.2 \\
\hline & $90-$ & 31 & 1.4 & 52 & 0.6 \\
\hline & Total & 2,172 & 100 & 8,129 & 100 \\
\hline Total time in follow-up ${ }^{c}$ & \multicolumn{3}{|l|}{7,287 years } & \multicolumn{2}{|c|}{34,330 years } \\
\hline Mean (SD) & \multicolumn{3}{|c|}{3.36 years (2.63 years) } & \multicolumn{2}{|c|}{4.22 years $(2.85$ years $)$} \\
\hline Median & \multicolumn{3}{|c|}{2.64 years $(0.02-12.74)$} & \multicolumn{2}{|c|}{3.78 years $(0.00-13.66)$} \\
\hline
\end{tabular}

In Fig. 2, the prevalence of psychotropic use among the female breast cancer patients treated with endocrine therapy is stratified for anxiolytics, hypnotics and sedatives and anti-depressants. Before the start of hormonal therapy, anxiolytics are most frequently prescribed, followed by hypnotics and sedatives, and anti-depressants. After the start of hormonal therapy, hypnotics and sedatives are prescribed most frequently. In the period 6 months before the start of hormonal therapy, the prevalences of anxiolytics and hypnotics and sedatives are significantly increased as compared with the use of these drugs longer before. For anxiolytics, the prevalence is 0.26 (95\% CI $0.24-0.27)$, and for hypnotics and sedatives, the prevalence is 0.24 (95\% CI
0.23-0.26) (Fig. 2). The prevalence of anti-depressant use is not increased.

\section{Discussion}

This large cohort study examined the use of psychotropic medication in women with breast cancer treated with endocrine therapy $(n=2,172)$ as compared to a group of matched women without cancer $(n=8,129)$. An increased prescribing of psychotropic medication in these women treated with endocrine therapy was shown, as was hypothesized. There was an increased start for anxiolytics (IRR
Table 2 Number of starters per year

A starter is defined as a woman who did not receive a prescription for this drug for at least 180 days. The database is relative stable since 1999

\begin{tabular}{lcllll}
\hline Year & Tamoxifen & Fulvestrant & Anastrazole & Letrozole & Exemestane \\
\hline 1994 & 8 & 0 & 0 & 0 & 0 \\
1995 & 34 & 0 & 0 & 0 & 0 \\
1996 & 54 & 0 & 0 & 0 & 0 \\
1997 & 87 & 0 & 12 & 0 & 0 \\
1998 & 158 & 0 & 40 & 1 & 0 \\
1999 & 218 & 0 & 37 & 5 & 0 \\
2000 & 218 & 0 & 36 & 23 & 6 \\
2001 & 194 & 0 & 37 & 15 & 7 \\
2002 & 197 & 0 & 57 & 22 & 27 \\
2003 & 165 & 0 & 96 & 32 & 32 \\
2004 & 159 & 0 & 88 & 54 & 45 \\
2005 & 148 & 13 & 148 & 67 & 75 \\
2006 & 149 & 21 & 123 & 83 & 53 \\
\hline
\end{tabular}


Table 3 Population at risk (n), incident cases (cases), incident rate (per 100,000 patient days) and IRR with 95\% CI for psychotropic medication

Treatment class IRR $(95 \% \mathrm{CI}) \quad$ Index (BC patients) (total $n=2,172)$

$n \quad$ Cases Days at risk $\quad$ IR $n \quad$ Cases $\quad$ Days at risk IR

Psychotropic medication

Anxiolytics

Hypnotics and sedatives

1,512

552

$1,848,232$

29.9

$1,651 \quad 580$

$2,102,023$

27,6

4,946

$2,703,233$

12,2

Anti-depressants

1,869

331

$B C$ breast cancer

2.07, 95\% CI 1.87-2.29), hypnotics and sedatives (IRR $2.59,95 \%$ CI 2.34-2.87) and anti-depressants (IRR 1.46, 95\% CI 1.28-1.65). Anxiolytics, hypnotics and sedatives were used for a longer time, whereas such a continuous increased use was not observed for anti-depressants. Among the women with anti-depressants, $20 \%$ received the drug combination of paroxetine and tamoxifen.

The increased percentage of women with breast cancer starting to use anxiolytics, hypnotics, sedatives and antidepressants indicates that in these women, there is an increased risk for psychological disorders. This is also mentioned in literature. Psychological problems are frequently mentioned in women treated for breast cancer, though there is a wide range in the estimated incidence $[8$, $9,15]$. To give an example, in a study including 1,083 women with a history of breast cancer (median 4 years after diagnosis) recruited through a population-based cancer registry (66\% response rate), 38\% of patients reported moderate to high anxiety, $22 \%$ moderate to high depression and $12 \%$ posttraumatic disorder [16]. In addition, two reviews on women with a history of breast cancer concluded that there is a substantial effect of adjuvant endocrine therapy on the health-related quality of life [9, 17]. Potential explanations for this impact on health-related quality of life are side effects of treatment due to premature menopause and mood disturbances [9]. High depression and anxiety levels in patients after breast cancer influence the coping with cancer and quality of life adversely [18].
We observed a statistically significant increase in the prescription of anti-depressants (IRR 1.46, 95\% CI 1.28 1.65). The prevalence of depression among early breast cancer women is estimated to be twice as many as that seen in the general population [19]. In addition, in our study, anxiolytics, hypnotics and sedatives were used for a longer time, whereas such a continuous increased use was not observed for anti-depressants. In literature, the increased prevalence of depression in women with a history of breast cancer is seen especially during the first year after diagnosis [19]. It can be stated that depression is undertreated among the breast cancer population [20]. On the other hand, problems of depression are not always treated with antidepressants, but also with anxiolytics, hypnotics and sedatives, and not all patients are willing to use medication for their depressed feelings. One of the most prescribed anti-depressants in this study was amitriptyline. In a low dose, amitriptyline can be prescribed for neuropathy as well as for sleep disturbances. As the prescribed dose distribution of amitriptyline was equal for women with breast cancer treated with endocrine therapy and for women without cancer (data not presented), we expected no effect on the presented incidence risk ratios. The relatively high prevalence of hypnotics (such as temazepam) and anti-depressants after start of endocrine treatment may also be related to the increased incidence of sleeping disorders due to hot flashes as one of the menopausal symptoms after starting endocrine treatment [21]. Improved sleeping quality on a combination

Table 4 Number of psychotropic drug users per group and specific drugs

\begin{tabular}{|c|c|c|c|c|c|}
\hline \multicolumn{2}{|c|}{ Anxiolytics (N05B) $n=552$} & \multicolumn{2}{|c|}{ Hypnotics and sedatives (N05C) $n=580$} & \multicolumn{2}{|c|}{ Anti-depressants (N06A) $n=331$} \\
\hline Oxazepam & 313 & Temazepam & 383 & Amitriptyline & 144 \\
\hline Diazepam & 159 & Lormetazepam & 48 & Paroxetine & 66 \\
\hline Lorazepam & 44 & Nitrazepam & 40 & Venlafaxine & 38 \\
\hline Alprazolam & 11 & Midazolam & 37 & Citalopram & 25 \\
\hline \multirow[t]{3}{*}{ Others } & 25 & Zopiclon & 34 & Mirtazapine & 19 \\
\hline & & Zolpidem & 19 & Others & 39 \\
\hline & & Others & 19 & & \\
\hline
\end{tabular}


Fig. 1 Prevalence of psychotropic medication use in index and reference groups. Medication groups included: anxiolytics, hypnotics and sedatives and anti-depressants

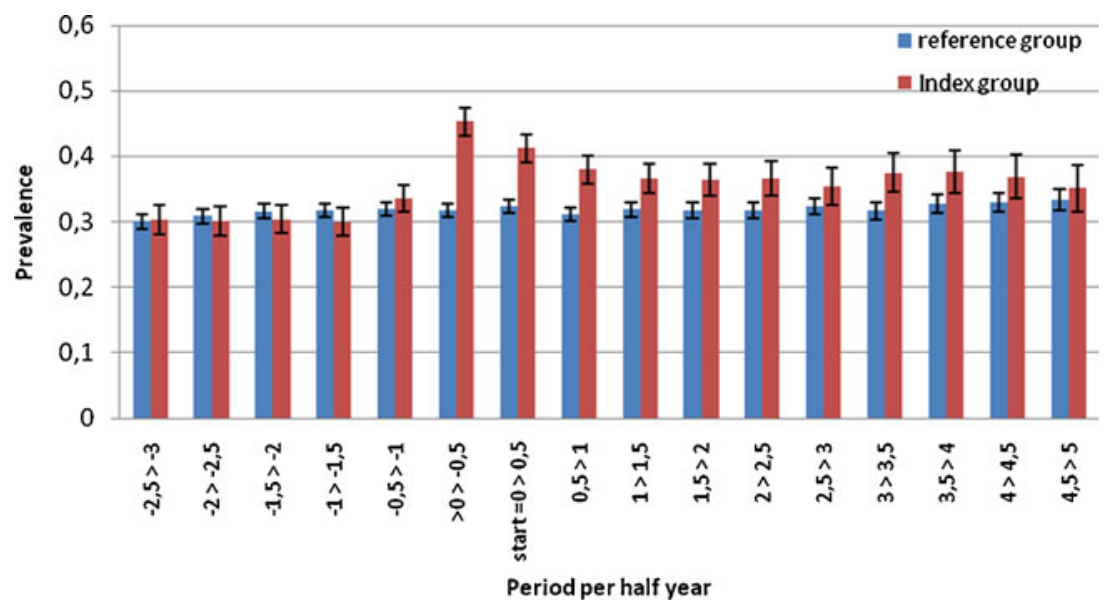

of venlafaxine and anti-hypnotics was recently found to contribute to improved quality of life in a similar group of breast cancer patients [22].

There are many reported side effects associated with use of psychotropic drugs. Side effects include but are not limited to: dry mouth, blurred vision, constipation, urinary retention, sedation, sleep disruption, weight gain or loss, headache, nausea, agitation, inability to achieve an orgasm, decreased libido and anxiety [clinicaldepression.com]. Longterm use of anxiolytics has been a cause for concern because of the possibility of dependency and other adverse consequences. Long-term effects of anxiolytics are the following: dependence, rebound anxiety, memory impairment and an increased risk of falling in elderly persons taking benzodiazepines [23]. Among the women with anti-depressants, $20 \%$ received the drug combination of paroxetine and tamoxifen. The use of paroxetine in this study is remarkable. A recent study showed that the use of paroxetine during tamoxifen treatment is associated with an increased risk of death from breast cancer [24]. Tamoxifen is a prodrug that is metab- olised to active metabolite endoxifen by the hepatic cytochrome P450 enzyme system. Paroxetine is a potent irreversible CYP 2D6 inhibitor that can prevent the metabolic activation of tamoxifen into the active metabolite endoxifen. Based on the study of Kelly et al., it is recommended to avoid this combination, and in case an anti-depressant is necessary, an anti-depressant with little or no inhibition of CYP 2D6 should be given. This is supported in the recently published guidelines on the use of comedication with tamoxifen [25]. However, because only short-term use of anti-depressants was found in this study, this may narrow the scope of this issue.

A strong point of the analysis presented here is that the used IADB database includes virtually complete prescription records for the patients covered by this database, except OTC drugs and drugs dispensed during a hospital stay [13]. As neither psychotropic medication nor endocrine therapies are OTC drugs in the Netherlands, this will not influence the outcomes. The estimated population size covered by the IADB.nl increased from 264.664 persons
Fig. 2 Prevalence of psychotropic medication per subgroup: anxiolytics, hypnotics and sedatives and anti-depressants among index group

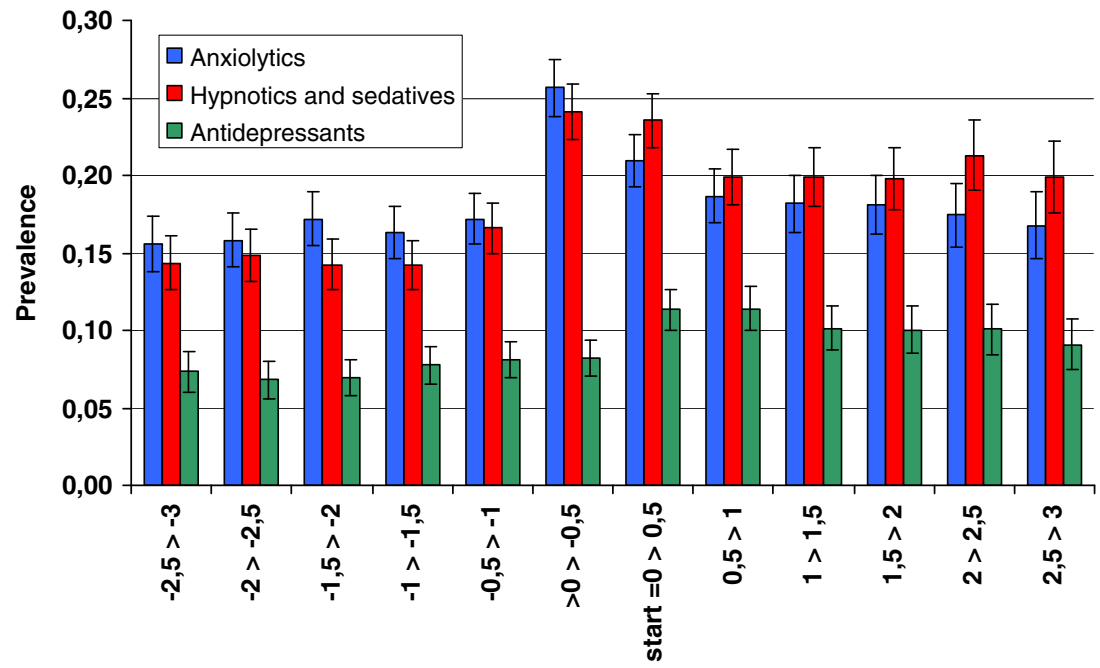

Period per half year 
in 1996 to approximately 500,000 persons in 1999 and has remained stable since. The women from the index group could be considered as representative of the Dutch population. In addition, the IADB.nl can be considered as a database that is representative for the Netherlands regarding drug prescriptions (manuscript in preparation). Regarding other prescriptions, there were no differences between women from the index group and women from the reference group.

A weak point is that there are no diagnoses of diseases in this database. As we have very strict guidelines for the prescription of endocrine treatment in the Netherlands [http:// www.oncoline.nl/], we were able to use a prescription of endocrine treatment as a proxy for a diagnosis of breast cancer. As a consequence, the following women were not included in our analysis: women with hormone-negative tumours, women with primary metastases and women with very small tumours. As our included sample is large, we do not expect that missing these groups might have a strong impact on the presented incidence risk ratios. In addition, we cannot analyse whether the increased use of psychotropic medication among breast cancer patients treated with endocrine treatment is related to the diagnosis of breast cancer or the related treatment. There is no formal evaluation of patients for mood disorder, anxiety or panic. We cannot be certain that the increased use of psychotropic medication is related to depression or anxiety. Benzodiazepines can also be prescribed for sleep and anti-depressants for pain.

In conclusion, in this large cohort study on breast cancer patients treated with endocrine therapy, we found an increased use of psychotropic medication, compared to the reference group. This may be related to the reported increased psychological distress in these patients. Anti-depressants were only prescribed for a short time. We do not know whether this is related to the condition of the patient with breast cancer or to the diagnostics of the prescriber. These data can contribute to an improved awareness of the impact of breast cancer (treatment) on quality of life and therefore potentially to the optimizing of support for these patients.

Conflicts of interest There are no conflicts or interests.

Open Access This article is distributed under the terms of the Creative Commons Attribution Noncommercial License which permits any noncommercial use, distribution, and reproduction in any medium, provided the original author(s) and source are credited.

\section{References}

1. Integraal Kanker Centrum. Available at http://www.ikcnet.nl. Accessed May 2009

2. Carlson RW, Hudis CA, Pritchard KI (2006) National Comprehensive Cancer Network Breast Cancer Clinical Practice Guide- lines in Oncology; American Society of Clinical Oncology Technology Assessment on the Use of Aromatase Inhibitors; St Gallen International Expert Consensus on the Primary Therapy of Early Breast Cancer. Adjuvant endocrine therapy in hormone receptor-positive postmenopausal breast cancer: evolution of NCCN, ASCO, and St Gallen recommendations. J Natl Compr Canc Netw 4:971-979

3. Goel S, Sharma R, Hamilton A, Beith J (2009) LHRH agonists for adjuvant therapy of early breast cancer in premenopausal women. Cochrane Database Syst Rev 4:CD004562

4. Klijn JG, Blamey RW, Boccardo F, Tominaga T, Duchateau L, Sylvester R (2001) Combined Hormone Agents Trialists' Group and the European Organization for Research and Treatment of Cancer. Combined tamoxifen and luteinizing hormone-releasing hormone (LHRH) agonist versus LHRH agonist alone in premenopausal advanced breast cancer: a meta-analysis of four randomized trials. J Clin Oncol 19:343-353

5. Ingle JN (2006) Adjuvant endocrine therapy for postmenopausal women with early breast cancer. Clin Cancer Res 12:1031s-1036s

6. Gibson L, Lawrence D, Dawson C, Bliss J (2009) Aromatase inhibitors for treatment of advanced breast cancer in postmenopausal women. Cochrane Database Syst Rev 4: CD003370

7. Early Breast Cancer Trialists' Collaborative Group (2005) Effects of chemotherapy and hormonal therapy for early breast cancer on recurrence and 15-year survival: an overview of the randomised trials. Lancet 365:1687-1717

8. Kornblith AB, Ligibel J (2003) Psychosocial and sexual functioning of survivors of breast cancer. Semin Oncol 39:799-813

9. Buijs C, de Vries EG, Mourits MJ, Willemse PH (2008) The influence of endocrine treatments for breast cancer on healthrelated quality of life. Cancer Treat Rev 34:640-655

10. IADB drug use research. Available at http://www.iadb.nl. Accessed Aug 2009

11. Farmacotherapeutische kompas. Available at http://www.fk.cvz.nl/ Accessed Jun 2009

12. WHO Collaborating Centre for Drugs Statistics Methodology. ATC/DDD index 2008. Available at http://www.whocc.no/ atcddd. Accessed May 2009

13. Monster TBM, Janssen WM, de Jong PE, de Jong-van den Berg LTW (2002) Pharmacy data in epidemiological studies: an easy to obtain and reliable tool. Pharmacoepidemiol Drug Saf 11:379-384

14. Jabaaij L (2002) Top 10: diagnoses en voorschrijven. Huisarts en Wetenschap 45:57 [In Dutch]

15. Lueboonthavatchai $P$ (2007) Prevalence and psychosocial factors of anxiety and depression in breast cancer patients. J Med Assoc Thai 90:2164-2174

16. Mehnert A, Koch U (2008) Psychological comorbidity and healthrelated quality of life and its association with awareness utilization, and need for psychosocial support in a cancer based sample of long-term breast cancer survivors. J Psychosom Res 64:383-391

17. Montazeri A (2008) Health-related quality of life in breast cancer patients: a bibliographic review of the literature from 1974 to 2007. J Exp Clin Cancer Res 27:32

18. Karakoyun-Celik O, Gorken I, Sahin S, Orcin E, Alanyali H, Kinay M (2010) Depression and anxiety levels in woman under follow-up for breast cancer: relationship to coping with cancer and quality of life. Med Oncol 27:108-113

19. Burgess C, Cornelius V, Love S, Graham J, Richards M, Ramirez A (2005) Depression and anxiety in women with early breast cancer: five year observational cohort study. Brit Med J 330:702 
20. Reich M, Lesur A, Perdrizet-Chevallier C (2008) Depression, quality of life and breast cancer: a review of the literature. Breast Cancer Res Treat 110:9-17

21. Buijs C, Mom CH, Willemse PH, Marike Boezen H, Maurer JM, Wymenga AN, de Jong RS, Nieboer P, de Vries EG, Mourits MJ (2009) Venlafaxine versus clonidine for the treatment of hot flashes in breast cancer patients: a doubleblind, randomized cross-over study. Breast Cancer Res Treat 115:573-580

22. Joffe H, Partridge A, Giobbie-Hurder A, Li X, Habin K, Goss P, Winer E, Garber J (2010) Augmentation of venlafaxine and selective serotonin reuptake inhibitors with zolpidem improves sleep and quality of life in breast cancer patients with hot flashes: a randomized, double-blind, placebo-controlled trial. Menopause $17: 889-891$

23. Uzun S, Kozumplik O, Jakovljević M, Sedić B (2010) Side effects of treatment with benzodiazepines. Psychiatr Danub 22:90-93

24. Kelly CM, Juurlink DN, Gomes T, Hua MD, Pritchard KI, Austin PC, Paszat LF (2010) Selective serotonin reuptake inhibitors and breast cancer mortality in women receiving tamoxifen: a population based cohort study. Brit Med J 340: c693. doi:10.1136/bmj

25. Sideras K, Ingle JN, Ames MM, Loprinzi CL, Mrazek DP, Black JL, Weinshilboum RM, Hawse JR, Spelsberg TC, Goetz MP (2010) Coprescription of tamoxifen and medications that inhibit CYP2D6. J Clin Oncol 28:2768-2776 